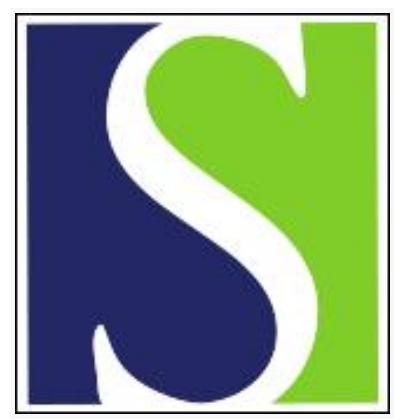

Scand J Work Environ Health 2003;29(2):85-93

https://doi.org/10.5271/sjweh.709

Issue date: Apr 2003

Time to pregnancy among the wives of Finnish greenhouse workers

by Sallmén M, Liesivuori J, Taskinen H, Lindbohm M-L, Anttila A, Aalto $L$, Hemminki K

Affiliation: Department of Epidemiology and Biostatistics, Finnish Institute of Occupational Health, Topeliuksenkatu 41 a A, FI-00250, Helsinki, Finland. Markku.Sallmen@ttl.fi

Refers to the following text of the Journal: $2000 ; 26(2): 131-136$

The following articles refer to this text: 2006;32(5):359-367;

2007;33(1):13-28

Key terms: exposure; fecundability; Finland; greenhouse worker; male; pregnancy delay; reproductive health; time to pregnancy; wife

This article in PubMed: www.ncbi.nlm.nih.gov/pubmed/12718493 


\title{
Time to pregnancy among the wives of Finnish greenhouse workers
}

\author{
by Markku Sallmén, PhD, ${ }^{1,2}$ Jyrki Liesivuori, PhD, ${ }^{3}$ Helena Taskinen, MD, ${ }^{4}$ Marja-Liisa Lindbohm, DrPH, ${ }^{1}$ \\ Ahti Anttila, PhD, ${ }^{1}$ Lea Aalto, ${ }^{1}$ Kari Hemminki, $M D^{5}$
}

\begin{abstract}
Sallmén M, Liesivuori J, Taskinen H, Lindbohm M-L, Anttila A, Aalto L, Hemminki K. Time to pregnancy among the wives of Finnish greenhouse workers. Scand J Work Environ Health 2003;29(2):85-93.
\end{abstract}

\begin{abstract}
Objectives This study investigated the possibility of men's work in greenhouses and their exposure to pesticides being associated with reduced fertility.

Methods A study on time to pregnancy was conducted among the families of Finnish male greenhouse employers and employees. Exposure to pesticides was assessed on the basis of questionnaire information and data gathered from the enterprises. Fecundability density ratios (FDR) for occupational exposure were calculated with discrete proportional hazards regression analyses.

Results After three mailings, 578 (43\%) couples participated. Fecundability was suggestively decreased for exposed greenhouse workers who were inefficiently protected, with FDR values of 0.67 [95\% confidence interval (95\% CI) 0.33-1.35], 0.92 [95\% CI 0.45-1.88] and 0.77 [95\% CI 0.46-1.29] for high exposure, moderate exposure and low exposure, respectively, as compared with unexposed greenhouse workers. The exposed men who efficiently used personal protective equipment were as fertile as the unexposed greenhouse workers. Exposure to pyrethroids (FDR 0.40, 95\% CI 0.19-0.85) was related to decreased fecundability. Suggestive associations were observed for organophosphates (FDR 0.70, 95\% CI 0.42-1.17) and carbamates (FDR 0.55, 95\% CI 0.27-1.11).

Conclusions The findings of the study provide limited support for the hypothesis that exposure to pesticides is associated with reduced fertility. The findings for pyrethroids, organophosphates, and carbamates can serve as a basis for a hypothesis for future studies.
\end{abstract}

Key terms fecundability, male exposure, pregnancy delay, reproductive health.

One of the first agents observed to be hazardous for male reproduction in humans was the nematocide DBCP ( $\mathrm{di}$ bromochloropropane) (1). Some other pesticides [ie, 2,4dichlorophenoxyacetic acid (2,4-D), ethylene dibromide (EDB), chlordecone (Kepone ${ }^{\mathrm{TM}}$ ), and carbaryl] have also been shown to cause adverse effects on semen quality (2-3). A Dutch study observed decreased fecundability among highly exposed fruit growers as compared with that of workers with low exposure (4), but three other studies found no association between exposure to pesticide and male fecundability (5-7). All in all, data on the effects of pesticide exposure on male fertility are scarce and difficult to compare due to exposure heterogeneity.

About 7000 permanent workers and 12000 temporary workers are employed in greenhouses in Finland. About $40 \%$ of the employees are men. The pesticides mainly used in Finnish greenhouses are herbicides and insecticides. Typical examples are carbamates (benomyl and thiophanate-methyl), dithiocarbamates (mancozeb and thiram), organochlorine compounds (dienochlor, dicofol, endosulfan, lindane), and sulfur (8-9). More

1 Department of Epidemiology and Biostatistics, Finnish Institute of Occupational Health, Helsinki, Finland.

2 Current affiliation: Epidemiology Branch, National Institute of Environmental Health Sciences, Research Triangle Park, North Carolina, United States.

3 Kuopio Regional Institute of Occupational Health, Kuopio, Finland, and Department of Pharmacology and Toxicology, University of Kuopio, Finland.

4 Department of Occupational Medicine, Finnish Institute of Occupational Health, Helsinki, Finland.

5 Karolinska Institutet, Department of Nutrition and Toxicology, Huddinge, Sweden.

Correspondence to: Dr Markku Sallmén, Department of Epidemiology and Biostatistics, Finnish Institute of Occupational Health, Topeliuksenkatu 41 a A, FIN-00250, Helsinki, Finland. [E-mail: Markku.Sallmen@ttl.fi] 
than half of the insecticides are organophosphates (dimethoate, dichlorvos, mevinphos, sulfotep). Carbamates (aldicarb, pirimicarb), pyrethroids (deltamethrin, permethrin), and nicotine are also used to control insects.

Pesticide concentrations in the air often exceed the occupational exposure limits in greenhouses when fumigated (10). Most insecticides, such as organophosphates, carbamates, and organochlorines, penetrate the skin easily. Most of the exposure occurs via the skin, depending on the application method (11). Some pesticides degrade on the leaves into more toxic compounds with even a stronger penetration capacity through the skin than their parent compounds. Workers are exposed to pesticides via inhalation during applications and to pesticide residues through the skin when they handle treated plants.

The aim of our study was to investigate whether the work of men in greenhouses and exposure to pesticides are associated with decreased fertility. Time to pregnancy was used as the measure of fertility (12). Valid data on time to pregnancy at a group level can be derived retrospectively, with a recall time of 14 years or more (13).

\section{Subjects and methods}

A study on time to pregnancy was conducted among Finnish male greenhouse employers and employees. The employers and employees who had been working in greenhouses at least one month in 1980-1990 were included. Data on male employees were collected from the files of the LEL Employment Pension Fund. All employees working in agriculture are insured under LEL. Data on greenhouse companies were obtained from employers' associations (1247 enterprises). Data on the identification codes of male employers and male family members, cultivated plants, and the pesticides used for each plant were collected with a questionnaire from the enterprises. The participation rate was 54\% in that inquiry. Due to missing information on male employers or family members the data covered only 478 male employers. Altogether data were obtained on 14733 employees and 419 employers who were born in 1930-1971 and were 19 to 50 years of age during the study period, 1980-1990.

Data on marital status, the wives of greenhouse workers and employers, and the addresses of the couples were collected from the Finnish Population Register Centre. The study was restricted to men in their first marriage at the time of the inquiry (May 1994). The men in their first marriage numbered 3622 among the employees and 303 among the employers. The pregnancies of the wives were identified from the nationwide database on medically diagnosed pregnancies in 1973-1990.
The database includes information on all births, spontaneous abortions, and induced abortions retrieved from the Hospital Discharge Register and records of hospital policlinics (outpatient clinics) in Finland (14). The number of wives having pregnancies was 2328 , and the total number of pregnancies was 4734 . Altogether $86 \%$ of the pregnancies were births, $9 \%$ were spontaneous abortions, $4 \%$ were induced abortions, and $0.6 \%$ were extrauterine pregnancies.

The inclusion criteria for couples and pregnancies were as follows: (i) the couple had to be married within 5 months after the estimated conception date of the pregnancy (376 exclusions), (ii) the couple had to have either a birth or a spontaneous abortion during the study period, 1980-1990 (349 exclusions), (iii) the wife had to be 18-39 years of age in the third month of the pregnancy (11 exclusions), (iv) the wife could not have had more than two pregnancies ending in spontaneous abortion in 1973-1990 (7 exclusions), and (v) the pregnancy should have started after the husband's entry into greenhouse work (180 exclusions). The study pregnancy was defined as the first eligible pregnancy (either a birth or a spontaneous abortion) during the study period. A total of 1407 couples met these criteria. In addition 72 couples were excluded because of a different address of the man and wife or the missing address of the couple.

Data on time to pregnancy and related factors were asked in questionnaires at two stages in 1994 to 1995. Data inquiry took place 4 to 15 years after the studied pregnancies. At the first stage, separate questionnaires were sent to 1335 men (1225 workers and 110 employers) and their wives to obtain data on the pregnancies, work histories, and personal habits. The potential confounders were selected on the basis of a priori knowledge on their association with fertility. The men were asked about their work history in greenhouses since 1975. There were detailed questions about the application of pesticides in the work area, the spraying of pesticides or work with the treated plants, the names of the pesticides used (the questionnaire included a list of the most common pesticides used in Finland), and the use of personal protective equipment. The men were asked to record these work conditions for each work period when necessary. Histories of the occurrence of solvents, welding work, exhausts, metal dusts or fumes, tobacco smoke, and work temperatures of $30^{\circ} \mathrm{C}$ or more were requested from both spouses. The wives were asked about their work history, work in greenhouses, pregnancy history, and the time required to get pregnant for each pregnancy that ended in a birth or a spontaneous abortion. The couples were asked about their possible fertility problems and medical consultations for such problems.

A total of 578 (43.3\%) couples participated after two reminders. Two of the participants did not report the 
study pregnancy, three had missing information on the dates of the pregnancies, and one refused further contact. Short-time workers (less than a year of work in greenhouses; participation rate 39\%) were less willing to participate, as compared with employers $(58 \%)$ or those who had worked for at least a year (53\%). Women who had experienced a spontaneous abortion were more passive (39\%) than women with a birth as the study pregnancy $(44 \%)$. In addition women with at least three pregnancies in the register (39\%) were less willing to participate than women with no more than two registered pregnancies $(46 \%)$. In addition to the 578 families, also 37 men $(2.8 \%)$ and 75 wives $(5.6 \%)$ participated in the first stage of the study only.

Another inquiry was restricted to the 572 families who participated in the first phase of the study. A second questionnaire was sent to wives to obtain detailed information on the time to pregnancy preceding the selected study pregnancy. Those who did not use any contraception at the time of conception were asked: "Did you get pregnant during the first menstrual cycle that occurred after you started sexual intercourse without contraception? During the second cycle? If not: How many cycles (or months) did it take to get pregnant? (years) months." The wives were asked about their last contraceptive method, the consumption of coffee and alcohol, and smoking habits preceding the pregnancy. Information on work and the occurrence or handling of pesticides or solvents at work was also requested.

Altogether 489 (85.5\%) wives participated in the second inquiry. Missing or contradictory information on time to pregnancy was complemented with phone calls $(\mathrm{N}=8)$ or questionnaires $(\mathrm{N}=13)$. The pregnancy was due to contraceptive failure in the case of 24 women. Only two $(0.4 \%)$ of the remaining women could not determine their time to pregnancy. Eight couples were excluded because of the wife's hormonal treatment for infertility or the study pregnancy had started through in vitro fertilization. The study was restricted to couples $(\mathrm{N}=588)$ with information on time to pregnancy from either the second $(\mathrm{N}=456)$ or the first $(\mathrm{N}=132)$ inquiry. In $522(88.8 \%)$ cases, also the man participated.

\section{Exposure assessment}

Exposure and the use of protective equipment were assessed for the beginning of the time-to-pregnancy period. The date when the time to pregnancy started was calculated using the dates from the medical records and the length of time to pregnancy allowing 80 days for spermatogenesis. An experienced occupational hygienist conducted the exposure assessment of male greenhouse workers without knowledge of the time to pregnancy. Exposure assessment was made for all the worktasks within the 12-month period following the onset of time to pregnancy, regardless of the length of time to pregnancy. Only the assessment for jobs during the time to pregnancy was used in the analyses, however. Exposure assessment was based on the following information: (i) questionnaire data, (ii) data on cultivated plants and used pesticides as reported by the employers, and (iii) results of previous studies on exposure conditions in Finnish greenhouses (9). The calendar months with pesticide exposure were taken into account in the assessment to reduce the misclassification of exposure. Male exposure to other occupational factors and female exposure were defined on the basis of the questionnaire information.

Both the likelihood and the level of exposure were assessed for exposure to pesticides in general. A greenhouse worker was classified as unexposed if he did not report any pesticide application or handling of treated plants. Workers were ranked as potentially exposed if their employers had offered information about pesticide applications in the company. The men who reported the application of pesticides or the handling of treated plants were regarded as exposed.

Exposure was rated as high if a person had applied pesticides at least once a week or had worked with treated plants on 3 days a week. The exposure was considered moderate if pesticides were applied two to three times a month or the treated plants were handled on 1 or 2 days a week. If a worker had sprayed pesticides only once a month or had worked with treated plants less than once a week, his exposure was considered low. In the analyses, potential exposure $(\mathrm{N}=5)$ was included in the low-exposure category. Use of protective equipment was efficient if a worker reported wearing appropriate protection whenever being in contact with pesticides or their residues. Protection was inefficient if a person did not use any protection or he wore protective equipment only sometimes or only during some tasks when exposure was possible. The exposure groups were decided before the data analysis was carried out.

Pesticides were classified into 11 categories: $1-6=$ insecticides: organophosphorous compounds, organochlorines, carbamates, nicotine, pyrethroids, organotin compounds (only 4 exposed workers); 7-9 = fungicides (bentzimidazoles, dithiocarbamates, other fungicides); $10=$ herbicides (all in the same group), and $11=$ plant growth regulators (all in the same group).

A total of $210(36 \%)$ men worked in greenhouses or gardens during the time to pregnancy, and 143 (24\%) were assessed as exposed to pesticides. Exposure was defined as high for 50 men, moderate for $28 \mathrm{men}$, and low for 65 men. Exposure could not be assessed for 8 men. Altogether 15 highly exposed men, and 11 and 32 men in the moderate and low exposure groups, respectively, did not use protective equipment efficiently. The association of male exposure to pesticides and 
Table 1. Time to pregnancy (TTP) distributions and unadjusted fecundability density ratios (FDR) according to men's work in greenhouses or gardens and potential confounders -21 proportional hazards models. ${ }^{a}(95 \% \mathrm{Cl}=95 \%$ confidence interval)

\begin{tabular}{|c|c|c|c|c|c|c|c|c|c|c|}
\hline & \multicolumn{8}{|c|}{ Time to pregnancy } & \multirow{3}{*}{ FDR } & \multirow{3}{*}{$95 \% \mathrm{Cl}$} \\
\hline & \multicolumn{2}{|c|}{ 1-2 months } & \multicolumn{2}{|c|}{ 3-6 months } & \multicolumn{2}{|c|}{ 7-12 months } & \multicolumn{2}{|c|}{$>12$ months } & & \\
\hline & $\mathrm{N}$ & $\%$ & N & $\%$ & $\mathrm{~N}$ & $\%$ & $\mathrm{~N}$ & $\%$ & & \\
\hline \multicolumn{11}{|l|}{ Male factors } \\
\hline \multicolumn{11}{|l|}{ Participation } \\
\hline No (only wife participated) & 46 & 69.7 & 15 & 22.7 & 3 & 4.6 & 2 & 3.0 & 1.0 & Reference \\
\hline Yes (both couples participated) & 293 & 56.1 & 143 & 27.4 & 40 & 7.7 & 46 & 8.8 & 0.69 & $0.53-0.92$ \\
\hline \multicolumn{11}{|l|}{ Work in greenhouses or gardens } \\
\hline $\begin{array}{l}\text { Yes } \\
\text { No (men in other occupations }\end{array}$ & 112 & 53.3 & 54 & 25.7 & 18 & 8.6 & 26 & 12.4 & 0.81 & $0.57-1.01$ \\
\hline or unemployed) & 227 & 60.1 & 104 & 27.5 & 25 & 6.6 & 22 & 5.8 & 1.0 & Reference \\
\hline \multicolumn{11}{|l|}{ Work in greenhouses or gardens } \\
\hline Yes $^{\mathrm{b}}$ & 112 & 53.3 & 54 & 25.7 & 18 & 8.6 & 26 & 12.4 & 0.84 & $0.62-1.15$ \\
\hline \multicolumn{11}{|l|}{$\begin{array}{l}\text { Exposure to pesticides (use of protective } \\
\text { equipment not considered) }\end{array}$} \\
\hline Not exposed & 31 & 52.5 & 15 & 25.4 & 8 & 13.6 & 5 & 8.5 & 1.0 & Reference \\
\hline Low exposure & 32 & 49.2 & 18 & 27.7 & 4 & 6.2 & 11 & 16.9 & 0.84 & $0.57-1.24$ \\
\hline Moderate exposure & 15 & 53.6 & 9 & 32.1 & 2 & 7.1 & 2 & 7.1 & 1.11 & $0.69-1.80$ \\
\hline High exposure & 29 & 58.0 & 9 & 18.0 & 4 & 8.0 & 8 & 16.0 & 0.92 & $0.61-1.39$ \\
\hline Unknown & 5 & 62.5 & 3 & 37.5 & - & 0 & - & 0 & 1.69 & $0.78-3.66$ \\
\hline \multicolumn{11}{|l|}{ Work in greenhouses or gardens } \\
\hline \multicolumn{11}{|l|}{$\begin{array}{l}\text { Exposure to pesticides (use of protective } \\
\text { equipment considered) }\end{array}$} \\
\hline $\begin{array}{l}\text { Not exposed } \\
\text { Exposed - no efficient protection }\end{array}$ & 31 & 52.5 & 15 & 25.4 & 8 & 13.6 & 5 & 8.5 & 1.0 & Reference \\
\hline Low exposure & 18 & 56.3 & 6 & 18.9 & 1 & 3.1 & 7 & 21.9 & 0.82 & $0.50-1.32$ \\
\hline Moderate exposure & 6 & 54.6 & 4 & 36.4 & - & 0 & 1 & 9.1 & 1.15 & $0.57-2.31$ \\
\hline High exposure & 7 & 46.7 & 4 & 26.7 & - & 0 & 4 & 26.7 & 0.69 & $0.36-1.32$ \\
\hline Exposed - protected efficiently & & & & & & & & & & \\
\hline Low exposure & 14 & 42.4 & 12 & 36.4 & 3 & 9.1 & 4 & 12.1 & 0.87 & $0.55-1.38$ \\
\hline Moderate exposure & 9 & 52.9 & 5 & 29.4 & 2 & 11.8 & 1 & 5.9 & 1.09 & $0.61-1.93$ \\
\hline High exposure & 22 & 62.9 & 5 & 14.3 & 4 & 11.4 & 4 & 11.4 & 1.05 & $0.67-1.65$ \\
\hline Unknown & 5 & 62.5 & 3 & 37.5 & - & 0 & - & 0 & 1.67 & $0.75-3.71$ \\
\hline Age at the beginning of TTP & & & & & & & & & & \\
\hline$<21$ years & 12 & 35.3 & 12 & 35.3 & 5 & 14.7 & 5 & 14.7 & 0.68 & $0.51-0.89$ \\
\hline $21-29$ years & 261 & 60.0 & 119 & 27.4 & 30 & 6.9 & 25 & 5.8 & 1.0 & Reference \\
\hline $30-44$ years & 66 & 55.5 & 27 & 22.7 & 8 & 6.7 & 18 & 18.2 & 0.73 & $0.58-0.92$ \\
\hline Smoking & & & & & & & & & & \\
\hline Nonsmokers & 99 & 57.9 & 53 & 30.0 & 9 & 5.3 & 10 & 5.9 & 1.0 & Reference \\
\hline Smokers & & & & & & & & & & \\
\hline $1-14$ cigarettes/day & 56 & 61.5 & 21 & 23.1 & 9 & 9.9 & 5 & 5.5 & 1.00 & $0.76-1.31$ \\
\hline$\geq 15$ cigarettes/day & 46 & 41.8 & 40 & 36.4 & 12 & 10.9 & 12 & 10.9 & 0.72 & $0.55-0.93$ \\
\hline Ex-smokers & 36 & 59.0 & 10 & 16.4 & 4 & 6.6 & 11 & 18.0 & 0.73 & $0.53-1.01$ \\
\hline Alcohol & & & & & & & & & & \\
\hline Nonusers & 64 & 60.4 & 30 & 28.3 & 7 & 6.6 & 5 & 4.7 & 1.0 & Reference \\
\hline Users & & & & & & & & & & \\
\hline$\leq 6$ drinks/week & 118 & 57.0 & 51 & 24.6 & 20 & 9.7 & 18 & 9.7 & 0.84 & $0.65-1.08$ \\
\hline$\geq 7$ drinks/week & 22 & 40.0 & 20 & 36.4 & 5 & 9.1 & 8 & 14.6 & 0.63 & $0.44-0.90$ \\
\hline Temperatures of $\geq 30^{\circ} \mathrm{C}$ at work & & & & & & & & & & \\
\hline 0 hours/week & 219 & 56.7 & 111 & 28.8 & 26 & 6.7 & 30 & 7.8 & 1.0 & Reference \\
\hline$\leq 2$ hours/week & 13 & 50.0 & 7 & 26.9 & 3 & 11.5 & 3 & 11.5 & 0.82 & $0.53-1.26$ \\
\hline$\geq 3$ hours/week & 31 & 47.0 & 16 & 24.2 & 9 & 13.9 & 10 & 15.2 & 0.72 & $0.54-0.96$ \\
\hline Female factors & & & & & & & & & & \\
\hline Age at the beginning of TTP & & & & & & & & & & \\
\hline$<21$ years & 44 & 51.7 & 27 & 31.8 & 6 & 7.1 & 8 & 9.4 & 0.86 & $0.67-1.11$ \\
\hline $21-29$ years & 258 & 59.6 & 115 & 26.6 & 31 & 7.2 & 29 & 6.7 & 1.00 & Reference \\
\hline $30-38$ years & 37 & 52.9 & 16 & 22.9 & 6 & 8.6 & 11 & 15.7 & 0.74 & $0.56-0.97$ \\
\hline Study pregnancy & & & & & & & & & & \\
\hline Birth & 316 & 58.1 & 146 & 26.8 & 40 & 7.4 & 42 & 7.7 & 1.0 & Reference \\
\hline Spontaneous abortion & 23 & 52.3 & 12 & 27.3 & 3 & 6.8 & 6 & 13.6 & 0.82 & $0.58-1.14$ \\
\hline Pregnancy order & & & & & & & & & & \\
\hline 1st pregnancy & 199 & 54.7 & 111 & 30.5 & 25 & 6.9 & 29 & 8.0 & 0.96 & $0.80-1.15$ \\
\hline 2nd and later pregnancies & 139 & 62.1 & 48 & 21.4 & 18 & 8.0 & 19 & 8.5 & 1.0 & Reference \\
\hline
\end{tabular}




\begin{tabular}{|c|c|c|c|c|c|c|c|c|c|c|}
\hline & \multicolumn{8}{|c|}{ Time to pregnancy } & \multirow{3}{*}{ FDR } & \multirow{3}{*}{$95 \% \mathrm{Cl}$} \\
\hline & \multicolumn{2}{|c|}{$1-2$ months } & \multicolumn{2}{|c|}{ 3-6 months } & \multicolumn{2}{|c|}{ 7-12 months } & \multicolumn{2}{|c|}{$>12$ months } & & \\
\hline & N & $\%$ & N & $\%$ & N & $\%$ & $\mathrm{~N}$ & $\%$ & & \\
\hline No previous births & 212 & 54.4 & 118 & 30.3 & 29 & 7.4 & 31 & 8.0 & 0.92 & $0.76-1.10$ \\
\hline Previous spontaneous abortion & 16 & 43.2 & 11 & 29.7 & 5 & 13.5 & 5 & 13.5 & 0.70 & $0.46-1.06$ \\
\hline $\begin{array}{l}\text { Previous spontaneous abortion } \\
\text { or extrauterine pregnancy } \\
\text { Last method of contraception }\end{array}$ & 20 & 44.4 & 13 & 28.9 & 6 & 13.3 & 6 & 13.3 & 0.69 & $0.47-1.00$ \\
\hline $\begin{array}{l}\text { Pill } \\
\text { Intrauterine device }\end{array}$ & $\begin{array}{l}69 \\
24\end{array}$ & $\begin{array}{l}46.3 \\
63.2\end{array}$ & $\begin{array}{r}54 \\
5\end{array}$ & $\begin{array}{l}36.2 \\
13.2\end{array}$ & $\begin{array}{r}19 \\
1\end{array}$ & $\begin{array}{r}12.8 \\
2.6\end{array}$ & $\begin{array}{l}7 \\
8\end{array}$ & $\begin{array}{r}4.7 \\
21.1\end{array}$ & $\begin{array}{l}1.17 \\
1.00\end{array}$ & $\begin{array}{l}0.90-1.52 \\
0.66-1.52\end{array}$ \\
\hline $\begin{array}{l}\text { Condom, rhythm method and } \\
\text { other unsafe method } \\
\text { None }\end{array}$ & $\begin{array}{r}103 \\
36\end{array}$ & $\begin{array}{l}67.3 \\
46.8\end{array}$ & $\begin{array}{l}34 \\
19\end{array}$ & $\begin{array}{l}22.2 \\
24.7\end{array}$ & $\begin{array}{r}5 \\
10\end{array}$ & $\begin{array}{r}3.3 \\
13.0\end{array}$ & $\begin{array}{l}11 \\
12\end{array}$ & $\begin{array}{r}7.2 \\
15.6\end{array}$ & $\begin{array}{l}1.53 \\
1.0\end{array}$ & $\begin{array}{l}1.17-1.99 \\
\text { Reference }\end{array}$ \\
\hline Marital status at the beginning of TTP & & & & & & & & & & \\
\hline $\begin{array}{l}\text { Unmarried } \\
\text { Married }\end{array}$ & $\begin{array}{r}62 \\
277\end{array}$ & $\begin{array}{l}46.3 \\
61.0\end{array}$ & $\begin{array}{r}49 \\
109\end{array}$ & $\begin{array}{l}36.6 \\
24.0\end{array}$ & $\begin{array}{r}9 \\
34\end{array}$ & $\begin{array}{l}6.7 \\
7.5\end{array}$ & $\begin{array}{l}14 \\
34\end{array}$ & $\begin{array}{r}10.5 \\
7.5\end{array}$ & $\begin{array}{l}1.0 \\
1.24\end{array}$ & $\begin{array}{l}\text { Reference } \\
1.01-1.53\end{array}$ \\
\hline Smoking & & & & & & & & & & \\
\hline $\begin{array}{l}\text { Nonsmokers } \\
\text { Smokers }\end{array}$ & 262 & 60.9 & 108 & 25.1 & 27 & 6.3 & 33 & 7.7 & 1.0 & Reference \\
\hline $\begin{array}{l}1-4 \text { cigarettes/day } \\
\geq 5 \text { cigarettes/day }\end{array}$ & $\begin{array}{l}11 \\
46\end{array}$ & $\begin{array}{l}47.8 \\
43.4\end{array}$ & $\begin{array}{r}7 \\
37\end{array}$ & $\begin{array}{l}30.4 \\
34.9\end{array}$ & $\begin{array}{r}2 \\
13\end{array}$ & $\begin{array}{r}8.7 \\
12.3\end{array}$ & $\begin{array}{r}3 \\
10\end{array}$ & $\begin{array}{r}13.0 \\
9.4\end{array}$ & $\begin{array}{l}0.75 \\
0.76\end{array}$ & $\begin{array}{l}0.48-1.16 \\
0.60-0.96\end{array}$ \\
\hline Coffee consumption & & & & & & & & & & \\
\hline $\begin{array}{l}\leq 4 \text { cups/day } \\
>4 \text { cups/day }\end{array}$ & $\begin{array}{r}184 \\
66\end{array}$ & $\begin{array}{l}57.0 \\
49.6\end{array}$ & $\begin{array}{l}92 \\
38\end{array}$ & $\begin{array}{l}28.5 \\
28.6\end{array}$ & $\begin{array}{l}25 \\
11\end{array}$ & $\begin{array}{l}7.7 \\
8.3\end{array}$ & $\begin{array}{l}22 \\
18\end{array}$ & $\begin{array}{r}6.8 \\
13.5\end{array}$ & $\begin{array}{l}1.0 \\
0.78\end{array}$ & $\begin{array}{l}\text { Reference } \\
0.63-0.98\end{array}$ \\
\hline Coffee consumption and smoking & & & & & & & & & & \\
\hline $\begin{array}{l}\text { Coffee } \leq 4 \text { cups/day } \\
\text { Nonsmokers }+ \text { smokers of } 1-4 \\
\text { cigarettes/day } \\
\text { Smokers of } \geq 5 \text { cigarettes/day }\end{array}$ & $\begin{array}{r}161 \\
23\end{array}$ & $\begin{array}{l}59.9 \\
42.6\end{array}$ & $\begin{array}{l}74 \\
18\end{array}$ & $\begin{array}{l}27.5 \\
33.3\end{array}$ & $\begin{array}{r}18 \\
7\end{array}$ & $\begin{array}{r}6.7 \\
13.0\end{array}$ & $\begin{array}{r}16 \\
6\end{array}$ & $\begin{array}{r}6.0 \\
11.1\end{array}$ & $\begin{array}{l}1.0 \\
0.63\end{array}$ & $\begin{array}{l}\text { Reference } \\
0.49-0.82\end{array}$ \\
\hline Coffee $>4$ cups/day & & & & & & & & & & \\
\hline $\begin{array}{l}\text { Nonsmokers or smokers of } \geq 5 \\
\text { cigarettes/day } \\
\text { Smokers of } \geq 5 \text { cigarettes/day }\end{array}$ & $\begin{array}{l}46 \\
20\end{array}$ & $\begin{array}{l}52.3 \\
44.4\end{array}$ & $\begin{array}{l}20 \\
18\end{array}$ & $\begin{array}{l}22.7 \\
40.0\end{array}$ & $\begin{array}{l}8 \\
3\end{array}$ & $\begin{array}{l}9.1 \\
6.7\end{array}$ & $\begin{array}{r}14 \\
4\end{array}$ & $\begin{array}{r}15.9 \\
8.9\end{array}$ & $\begin{array}{l}0.69 \\
1.23\end{array}$ & $\begin{array}{l}0.53-0.90 \\
0.83-1.84\end{array}$ \\
\hline Alcohol & & & & & & & & & & \\
\hline $\begin{array}{l}\text { Nonusers } \\
\text { Users }\end{array}$ & 129 & 63.2 & 49 & 24.0 & 14 & 6.9 & 12 & 5.9 & 1.0 & Reference \\
\hline $\begin{array}{l}\leq 2 \text { or unknown drinks/week } \\
\geq 3 \text { drinks/week }\end{array}$ & $\begin{array}{r}161 \\
22\end{array}$ & $\begin{array}{l}55.3 \\
42.3\end{array}$ & $\begin{array}{l}83 \\
17\end{array}$ & $\begin{array}{l}28.5 \\
32.7\end{array}$ & $\begin{array}{r}22 \\
4\end{array}$ & $\begin{array}{l}7.6 \\
7.7\end{array}$ & $\begin{array}{r}25 \\
9\end{array}$ & $\begin{array}{r}8.6 \\
17.3\end{array}$ & $\begin{array}{l}0.85 \\
0.61\end{array}$ & $\begin{array}{l}0.70-1.03 \\
0.44-0.86\end{array}$ \\
\hline $\begin{array}{l}\text { Employment and greenhouse or } \\
\text { garden work }\end{array}$ & & & & & & & & & & \\
\hline Not employed & 59 & 71.1 & 19 & 22.9 & 2 & 2.4 & 3 & 3.6 & 1.0 & Reference \\
\hline Employed & 191 & 51.2 & 111 & 29.8 & 34 & 9.1 & 37 & 9.9 & 0.61 & $0.47-0.80$ \\
\hline In a greenhouse or garden & 16 & 53.3 & 11 & 36.7 & 2 & 6.7 & 1 & 3.3 & 1.20 & $0.88-1.64$ \\
\hline Sprayed pesticides herself & 7 & 43.8 & 3 & 18.8 & 1 & 6.3 & 5 & 31.3 & 0.48 & $0.25-0.94$ \\
\hline
\end{tabular}

a A category of missing information was included in the model when necessary.

${ }^{b}$ Reference: men from other occupations or unemployed men.

fertility was assessed for the 58 inefficiently protected and 85 efficiently protected workers with exposure, compared with 59 greenhouse or garden workers without any exposure.

\section{Statistical analysis}

Time-to-pregnancy data were analyzed with a discrete proportional hazards regression analysis (15) using the SAS PHREG procedure and EXACT handling of ties (SAS Institute, Cary, NC, USA). Time-to-pregnancy categories of more than 13 cycles were combined into one category to exclude the possible effects of medical treatment for infertility, and because of the sparseness of the data beyond this point. The outcome parameter is a hazard ratio. Since pregnancy is a desired outcome, we used the phrase "fecundability density ratio" (FDR) in place of hazard ratio (16). FDR values below unity reflect reduced fertility.

The covariates were selected for the final model on the basis of the association with fecundability (FDR $\leq 0.7$ or FDR $\geq 1.4$ or P-value $<0.1$ ). The selection was performed starting with female age and reproductive factors, followed by personal habits, occupational factors, and male factors. Covariates for male age, smoking, use of alcohol, and exposure to temperatures of $30^{\circ} \mathrm{C}$ or more were not associated with fecundability in the multivariate models and were thus not included in the final model. It was checked that their inclusion in the model did not change the findings for male pesticide 
exposure. An interaction variable for female coffee use and smoking was included in the final model on the basis of a recent finding (17).

\section{Results}

The distributions and unadjusted FDR values are presented in table 1 (on pages $88 \& 89$ ). Among the female factors, older age, no contraceptive use preceding time to pregnancy, smoking, consumption of coffee and alcohol, employment, and spraying pesticides were associated with prolonged time to pregnancy. Among the male factors, older age, smoking, consumption of alcohol, and work in temperatures of $30^{\circ} \mathrm{C}$ or more were

Table 2. Adjusted fecundability density ratio (FDR) by exposure to pesticides-proportional hazards model. ${ }^{\text {a }}(95 \% \mathrm{Cl}=95 \%$ confidence interval, TTP = time to pregnancy)

\begin{tabular}{|c|c|c|c|}
\hline & N & FDR & $95 \% \mathrm{Cl}$ \\
\hline \multicolumn{4}{|l|}{ Male factors } \\
\hline \multicolumn{4}{|l|}{ Participation ${ }^{b}$} \\
\hline Yes & 522 & 0.75 & $0.50-1.12$ \\
\hline Work at greenhouses or gardens ${ }^{c}$ & 210 & 0.86 & $0.62-1.19$ \\
\hline \multicolumn{4}{|l|}{ Exposure to pesticides } \\
\hline \multicolumn{4}{|l|}{ No efficient protection } \\
\hline Low exposure & 32 & 0.77 & $0.46-1.29$ \\
\hline Medium exposure & 11 & 0.92 & $0.45-1.88$ \\
\hline High exposure & 15 & 0.67 & $0.33-1.35$ \\
\hline \multicolumn{4}{|l|}{ Protected efficiently } \\
\hline Low exposure & 33 & 0.82 & $0.51-1.34$ \\
\hline Medium exposure & 17 & 1.15 & $0.63-2.11$ \\
\hline High exposure & 35 & 0.94 & $0.58-1.52$ \\
\hline Unknown & 8 & 2.00 & $0.86-4.64$ \\
\hline Not exposed & 59 & 1.0 & Reference \\
\hline \multicolumn{4}{|l|}{ Female factors } \\
\hline Married at the beginning of TTP & 454 & 1.33 & $1.06-1.68$ \\
\hline Age $\geq 30$ years & 70 & 0.67 & $0.49-0.92$ \\
\hline First pregnancy & 364 & 0.88 & $0.71-1.09$ \\
\hline \multicolumn{4}{|l|}{ Last method of contraception ${ }^{d}$} \\
\hline No & 77 & 0.74 & $0.54-0.99$ \\
\hline $\begin{array}{l}\text { Condom, rhythm method, and } \\
\text { other unsafe method }\end{array}$ & 153 & 1.33 & $1.06-1.68$ \\
\hline \multicolumn{4}{|l|}{ Smoking } \\
\hline 1-4 cigarettes/day & 23 & 0.70 & $0.45-1.11$ \\
\hline$\geq 5$ cigarettes/day & 106 & 0.68 & $0.50-0.91$ \\
\hline \multicolumn{4}{|l|}{ Coffee $>4$ cups/day } \\
\hline $\begin{array}{l}\text { Nonsmokers }+ \text { smokers of } 1-4 \\
\text { cigarettes/day }\end{array}$ & 88 & 0.75 & $0.57-0.99$ \\
\hline Smokers of $\geq 5$ cigarettes/day & 45 & 1.42 & $0.93-2.18$ \\
\hline \multicolumn{4}{|l|}{ Alcohol } \\
\hline$\leq 2$ or unknown drinks/week & 291 & 0.82 & $0.67-1.01$ \\
\hline$\geq 3$ drinks/week & 52 & 0.66 & $0.46-0.96$ \\
\hline \multicolumn{4}{|l|}{ Employment ${ }^{\mathrm{e}}$} \\
\hline Employed & 373 & 0.69 & $0.52-0.91$ \\
\hline Work in greenhouses or gardens & 69 & 1.19 & $0.85-1.68$ \\
\hline Sprayed pesticides herself & 16 & 0.51 & $0.26-1.01$ \\
\hline
\end{tabular}

${ }^{a} \mathrm{~A}$ category of missing information was included in the model when necessary.

b Reference: only wife participated.

c Reference: no work in greenhouses or gardens.

d Reference: intrauterine device, pills.

e Reference: not employed. related to lower fertility. The families in which both couples participated in the study were less fertile than those in which only the wife participated. The wife's participation in the second inquiry was related to reduced fertility (crude FDR 0.78 , 95\% confidence interval (95\% CI) 0.63-0.96).

The prevalence of men working in greenhouses or gardens decreased over time- $55 \%, 32 \%$, and $17 \%$ of the men working in that branch in 1979-1984, 19851987, and 1988-1989, respectively. Fecundability was slightly decreased for greenhouse and garden workers as compared with that of men from other occupations or unemployed men (unadjusted FDR 0.81, 95\% CI 0.57-1.01) when pesticide exposure was not considered (table 1 , on pages $88 \& 89$ ).

Fecundability was slightly decreased for the exposed greenhouse workers who were inefficiently protected [adjusted FDR 0.67 (95\% CI 0.33-1.35), 0.92 (95\% CI $0.45-1.88$ ) and 0.77 (95\% CI 0.46-1.29) for high exposure, moderate exposure and low exposure, respectively] as compared with that of the unexposed greenhouse workers (table 2). The exposed men who used personal protective equipment efficiently were as fertile as the unexposed greenhouse workers.

Subgroup analyses were conducted for wives' participation in the second inquiry and for background variables that showed differences in participation rates (table 3 on page 91). The results were essentially unchanged among the men who had worked in greenhouses for at least 4 months during the study period, among the pregnancies ending in births, and according to the wives' participation. Male exposure to pesticides was associated with decreased fecundability among primiparous families [FDR 0.31 (95\% CI $0.10-0.99)$ and 0.43 (95\% CI 0.22-0.81) for high and lower exposure, respectively]. However, male exposure to pesticides was not related to fecundability among parous wives.

Fertility was examined according to the type of pesticides used (table 4 on page 92). Exposure to pyrethroids (FDR $0.40,95 \%$ CI $0.19-0.85$ ) was related to decreased fecundability. Suggestive associations were observed for organophosphates (FDR $0.70,95 \%$ CI $0.42-1.17$ ) and carbamates (FDR $0.55,95 \%$ CI $0.27-$ 1.11).

\section{Discussion}

Fecundability was suggestively reduced among the exposed men without efficient personal protection as compared with that of the unexposed greenhouse workers. The exposed men who wore personal protective equipment efficiently were as fertile as the unexposed workers. The main result was based on only 15 highly 
Table 3. Adjusted fecundability density ratios (FDR) for men's exposure to pesticides with modifications of the basic model一five proportional hazards regression models. ${ }^{\text {a }}(95 \% \mathrm{Cl}=95 \%$ confidence interval).

\begin{tabular}{|c|c|c|c|}
\hline & FDR & $95 \% \mathrm{Cl}$ & Modification \\
\hline \multicolumn{3}{|l|}{ Model 1} & Only employers and workers who had worked at least 4 \\
\hline \multicolumn{4}{|l|}{$\begin{array}{l}\text { Exposure to pesticides } \\
\text { Not efficiently protected }\end{array}$} \\
\hline Low-to-moderate exposure & 0.93 & $0.54-1.60$ & \\
\hline High exposure & 0.73 & $0.31-1.71$ & \\
\hline Protected efficiently & 1.07 & $0.66-1.71$ & \\
\hline \multicolumn{3}{|l|}{ Model 2} & Restricted to pregnancies ending in births $(\mathrm{N}=544)$ \\
\hline \multicolumn{4}{|l|}{ Exposure to pesticides } \\
\hline \multicolumn{4}{|l|}{ Not efficiently protected } \\
\hline Low-to-moderate exposure & 0.83 & $0.52-1.32$ & \\
\hline High exposure & 0.57 & $0.27-1.22$ & \\
\hline Protected efficiently & 0.85 & $0.57-1.26$ & \\
\hline Model 3 & & & Restricted to primiparous women ( $N=391)$ \\
\hline Work in greenhouses or gardens & 1.35 & $0.85-2.15$ & \\
\hline \multicolumn{4}{|l|}{ Exposure to pesticides } \\
\hline \multicolumn{4}{|l|}{ Not efficiently protected } \\
\hline Low-to-moderate exposure & 0.43 & $0.22-0.81$ & \\
\hline High exposure & 0.31 & $0.10-0.99$ & \\
\hline Protected efficiently & 0.59 & $0.34-1.01$ & \\
\hline Model 4 & & & Restricted to women with previous children $(\mathrm{N}=197)$ \\
\hline Work in greenhouses or gardens & 0.61 & $0.36-1.02$ & \\
\hline \multicolumn{4}{|l|}{ Exposure to pesticides } \\
\hline \multicolumn{4}{|l|}{ Not efficiently protected } \\
\hline Low-to-moderate exposure & 1.24 & $0.58-2.64$ & \\
\hline High exposure & 1.48 & $0.53-4.08$ & \\
\hline Protected efficiently & 1.32 & $0.71-2.47$ & \\
\hline Model 5 & & & Wife participated in the second stage of the inquiry $(\mathrm{N}=456)$ \\
\hline \multicolumn{4}{|l|}{ Exposure to pesticides } \\
\hline \multicolumn{4}{|l|}{ Not efficiently protected } \\
\hline Low-to-moderate exposure & 0.86 & $0.52-1.42$ & \\
\hline High exposure & 0.59 & $0.28-1.25$ & \\
\hline Protected efficiently & 0.83 & $0.55-1.26$ & \\
\hline
\end{tabular}

a Adjusted for male participation, female age, previous pregnancies, last contraceptive method, marital status, smoking, interaction between drinking coffee and smoking, use of alcohol, employment, work in greenhouses or gardens, spraying of pesticides, and missing information.

exposed men; this low number, together with the low participation rate, weakens the conclusions drawn from this study.

Our findings are in accordance with the observations of two Dutch studies $(4,18)$. In the first study, fertility was reduced in the families of exposed fruit growers (4). In the other study (18), male exposure to pesticides was related to reduced fertilization capacity. However, exposure to pesticides was associated with an improved implantation rate in the same in vitro fertilization data (19). Other studies do not suggest an association between male exposure and fecundability (5-7).

Our study was a synthetic prospective study conditional on pregnancy (20). The first time to pregnancy after the husband's entry into greenhouse work was followed. This design has two major advantages. First, the selected time to pregnancy is the most likely to have occurred during male exposure. This point is essential if short-time workers are considered. Second, the design is less prone to the so-called time trend bias, which may be prominent in retrospective studies on time to pregnancy, if the exposure under study has changed over calendar time (21-22). The potential for time-trend bias and truncation bias was also reduced since the comparisons were made among the greenhouse workers. The prevalence of greenhouse or garden work declined during the study period. Therefore, the suggestive finding of decreased fecundability observed for male work in greenhouses overestimates the true association. However, there was no clear time trend in the prevalence of pesticide exposure within the greenhouse workers, and the findings for male pesticide exposure are more reliable. In addition, the potential for the confounding effect of work at high temperatures or humidity was reduced because of the comparison within the greenhouse workers.

The strengths of the exposure assessment were as follows: (i) data on male exposure were collected 
Table 4. Adjusted fecundability density ratios (FDR) by the type of pesticide exposure-10 proportional hazards models. ${ }^{a}(95 \% \mathrm{Cl}=$ $95 \%$ confidence interval)

\begin{tabular}{|c|c|c|c|}
\hline Type of pesticide & $\mathrm{N}$ & FDR & $95 \% \mathrm{Cl}$ \\
\hline \multicolumn{4}{|l|}{ Organophosphates } \\
\hline Not protected efficiently & 32 & 0.70 & $0.42-1.17$ \\
\hline Protected efficiently & 66 & 0.92 & $0.61-1.39$ \\
\hline \multicolumn{4}{|l|}{ Organochlorines } \\
\hline Not protected efficiently & 15 & 1.04 & $0.54-2.02$ \\
\hline Protected efficiently & 40 & 1.09 & $0.70-1.70$ \\
\hline \multicolumn{4}{|l|}{ Carbamates } \\
\hline Not protected efficiently & 15 & 0.55 & $0.27-1.11$ \\
\hline Protected efficiently & 38 & 1.04 & $0.65-1.66$ \\
\hline \multicolumn{4}{|l|}{ Nicotine } \\
\hline Not protected efficiently & 10 & 1.16 & $0.53-2.54$ \\
\hline Protected efficiently & 18 & 0.84 & $0.47-1.52$ \\
\hline \multicolumn{4}{|l|}{ Pyrethroids } \\
\hline Not protected efficiently & 15 & 0.40 & $0.19-0.85$ \\
\hline Protected efficiently & 31 & 1.02 & $0.62-1.66$ \\
\hline \multicolumn{4}{|l|}{ Benzimidazoles } \\
\hline Not protected efficiently & 22 & 0.87 & $0.49-1.53$ \\
\hline Protected efficiently & 46 & 0.97 & $0.62-1.52$ \\
\hline \multicolumn{4}{|l|}{ Dithiocarbamates } \\
\hline Not protected efficiently & 11 & 1.68 & $0.80-3.54$ \\
\hline Protected efficiently & 23 & 0.73 & $0.42-1.28$ \\
\hline \multicolumn{4}{|l|}{ Miscellaneous fungicides } \\
\hline Not protected efficiently & 26 & 0.70 & $0.40-1.20$ \\
\hline Protected efficiently & 52 & 1.10 & $0.71-1.70$ \\
\hline \multicolumn{4}{|l|}{ Herbicides } \\
\hline Not protected efficiently & 11 & 0.67 & $0.31-1.48$ \\
\hline Protected efficiently & 17 & 0.67 & $0.36-1.26$ \\
\hline \multicolumn{4}{|l|}{ Plant growth regulators } \\
\hline All ${ }^{b}$ & 16 & 0.92 & $0.51-1.65$ \\
\hline
\end{tabular}

a Adjusted for male participation, female age, previous pregnancies, last contraceptive method, marital status, smoking, interaction between drinking coffee and smoking, use of alcohol, employment, work in greenhouses or gardens, spraying of pesticides, and missing information.

${ }^{\mathrm{b}} \mathrm{All}=3$ persons not protected efficiently and 13 persons protected efficiently.

independently of the studied outcome, (ii) exposure assessment was conducted by an experienced occupational hygienist without knowledge of the time to pregnancy, and (iii) seasonal variation in the use of pesticides was considered. The shortcomings were as follows: (i) exposure assessment was based mainly on self-reports of the work conditions and exposure and (ii) no specific data on occupational hygiene measurements from the companies or biomonitoring of the workers were available. Proper protective clothing is effective in decreasing the exposure of workers during and after applications (11). The finding that the association was enhanced among those who did not use personal protection efficiently makes the results more plausible.

It is difficult to attribute the possible risk to any specific pesticide. Simultaneous exposure to several types of pesticides was prevalent in our data. In addition, only few workers were exposed to individual pesticides or were highly exposed to different types of pesticides.
Therefore, only the findings of any exposure to the types of pesticides are presented.

Many Finnish greenhouses are family enterprises. Therefore, also a proportion of the wives was exposed to pesticides. Our findings, adjusted for male exposure, suggest that heavy female exposure, characterized by the women spraying pesticides, may be associated with reduced fertility. The finding is in accordance with that of a recent Danish study (23) in which female flower greenhouse workers handling treated cultures, spraying pesticides, and working without gloves had reduced fecundability.

The main shortcoming of our study was the low participation rate (43\%). Participation was related to job seniority and the number of pregnancies in the medical records. The main comparison was made between exposed and unexposed greenhouse workers. Among them the participation was slightly higher (49\%) because long-time workers and employers were overrepresented in these groups. The findings were virtually unchanged in the analysis that was restricted to more permanent workers. This observation is not suggestive of selection bias.

Separate questionnaires were sent to the men and their wives. The couples were not aware that the focus was on the first time to pregnancy after the husband's entry into greenhouse work. In the first questionnaire, we collected detailed information on up to five work periods in greenhouses. Information on time to pregnancy for all the pregnancies ending in births or spontaneous abortions was collected independently from the wives. The eligible study pregnancy was defined on the basis of medical records, work pension information, and data collected from the employers. It does not seem plausible that the couples could connect the husband's exposure status or the use of efficient protection and the time taken to conceive. Because of these methodological aspects, we believe that the potential for response bias is rather low.

Men's exposure to pesticides was associated with reduced fertility among primiparous wives, whereas among parous wives the association was towards improved fertility. It is not likely that response bias alone could cause such a big difference in the findings. It has been suggested that one should always use the subset of first-time pregnancies in a separate analysis when estimating the exposure-effect association (24). If the effect measure among primiparous women differs much from the overall measure, bias related to selection forces may well be the explanation. This may have been the case in our study as well. However, it is difficult to assess the direction of this potential bias because the first period of time to pregnancy after the husband's entry into greenhouse work was followed. 
The wives who responded to the first inquiry but not to the second (data on time to pregnancy from the first inquiry) were more fertile than those responding to both inquiries. The association between pesticide exposure and fertility was, however, similar in both phases of the study. This observation does not suggest selection bias in relation to the studied association. However, this finding is an indication of the poor comparability of differently collected data on time to pregnancy (25-27).

The findings of our study provide limited support for the hypothesis that men's work in greenhouses and exposure to pesticides are associated with reduced fertility. The findings on the exposure to pyrethroids, carbamates, and organophosphates can serve as a basis for a hypothesis for future studies.

\section{Acknowledgments}

The study was supported by a grant from the Finnish Work Environment Fund. We thank Lars Nylund for the linguistic revision of the Swedish questionnaires and Ritva Järnström, Maila Kämppi and Jorma Seitsamo for their technical help.

\section{References}

1. Whorton MD, Krauss RM, Marshall S, Milby TH. Infertility in male pesticide workers. Lancet 1977;2:1259-61.

2. Mattison DR, Bogumil RJ, Chapin R, Hatch M, Hendrickx A, Jarrell J, et al. Reproductive effects of pesticides. In: SR Baker, CF Wilkinson, editors. The effects of pesticides on human health. Princeton (NJ): Princeton Scientific Publishing Co; 1990. p 297-389.

3. Lähdetie J. Occupation- and exposure-related studies on human sperm. J Occup Environ Med 1995;37:922-30.

4. De Cock J, Westweer K, Heederik D, Velde E, Kooij R. Time to pregnancy and occupational exposure to pesticides in fruit growers in the Netherlands. Occup Environ Med 1994;51:693-9.

5. Larsen SB, Joffe M, Bonde JP, ASCLEPIOS study group. Time to pregnancy and exposure to pesticides in Danish farmers. Occup Environ Med 1998;55:278-83.

6. Thonneau P, Abell A, Larsen SB, Bonde JP, Joffe M, Clavert A, et al. Effects of pesticide exposure on time to pregnancy: results of a multicenter study in France and Denmark. Am J Epidemiol 1999;150:157-63.

7. Curtis KM, Savitz DA, Weinberg CR, Arbuckle TE. The effect of pesticide exposure on time to pregnancy. Epidemiology 1999;10:112-7.

8. Manninen A. Torjunta-aineet [Pesticides]. Helsinki:
Työterveyslaitos ja Työsuojelurahasto; 1991. Altisteet työssä, no 25.

9. Kangas J, Manninen A, Liesivuori J. Occupational exposure to pesticides in Finland. Int J Environ Anal Chem 1995;58: 423-9.

10. Liesivuori J, Liukkonen S, Pirhonen P. Reentry intervals after pesticide application in greenhouses. Scand J Work Environ Health 1988;14 Suppl 1:35-6.

11. Kangas J, Laitinen S, Jauhiainen A, Savolainen K. Exposure of sprayers and plant handlers to mevinphos in Finnish greenhouses. Am Ind Hyg Assoc J 1993;54:150-7.

12. Baird DD, Wilcox AJ, Weinberg CR. Use of time to pregnancy to study environmental exposures. Am J Epidemiol 1986; 124:470-80.

13. Joffe M, Villard L, Li Z, Plowman R, Vessey M. Long term recall of time-to-pregnancy. Fertil Steril 1993;60:99-104.

14. Lindbohm ML, Hemminki K. Nation-wide data base on medically diagnosed spontaneous abortions in Finland. Int J Epidemiol 1988;17:568-73.

15. Kalbfleisch JD, Prentice RL. The statistical analysis of failure-time data. New York (NY): Wiley; 1980.

16. Sallmén M, Lindbohm M-L, Anttila A, Kyyrönen P, Taskinen $\mathrm{H}$, Hemminki K. Time to pregnancy among the wives of men exposed to organic solvents. Occup Environ Med 1998; 55:24-30.

17. Stanton CK, Gray RH. Effects of caffeine consumption on delayed conception. Am J Epidemiol 1995;142:1322-9.

18. Tielemans E, van Kooij R, te Velde ER, Burdorf A, Heederik D. Pesticide exposure and decreased fertilisation rates in vitro. Lancet 1999;354:484-5.

19. Tielemans E, van Kooij R, Looman C, Burdorf A, te Velde ER, Heederik D. Paternal occupational exposures and implantation rates after IVF. Fertil Steril 2000;74:690-5.

20. Weinberg CR, Baird DD, Wilcox AJ. Sources of bias in studies of time to pregnancy. Stat Med 1994;13:671-81.

21. Weinberg CR, Baird DD, Rowland AS. Pitfalls inherent in retrospective time-to-event studies: the example of time to pregnancy. Stat Med 1993;12:867-79.

22. Sallmén M, Lindbohm M-L, Anttila A, Taskinen H, Hemminki K. Time to pregnancy among the wives of men occupationally exposed to lead. Epidemiology 2000;11:141-7.

23. Abell A, Juul S, Bonde JPE. Time to pregnancy among female greenhouse workers. Scand J Work Environ Health 2000;26:131-6.

24. Olsen J. Options in making use of pregnancy history in planning and analysing studies of reproductive failure. J Epidemiol Community Health 1994;48:171-4.

25. Sallmén M. Fertility in UK compared with Finland [comment]. Lancet 1996;348:616. Comment on: Joffe M. Lancet 1996;347:1519-22.

26. Tuntiseranee $\mathrm{P}$, Olsen J, Chongsuvivatwong V, Limburata S. Fecundity in Thai and European regions: results based on waiting time to pregnancy. Hum Reprod 1998;13:471-7.

27. Juul S, Karmaus W, Olsen J, The European Infertility and Subfecundity Study Group. Regional differences in waiting time to pregnancy: pregnancy-based surveys from Denmark, France, Germany, Italy and Sweden. Hum Reprod 1999; $14: 1250-4$.

Received for publication: 19 April 2002 